\title{
ACCELERATION GAP FOR THE RELATIVISTIC-KLYSTRON TWO-BEAM ACCELERATOR *
}

\author{
E. Zaidman, S. Douglass, and T. Godlove, FM Technologies, Inc., Fairfax, VA, USA
}

\begin{abstract}
We describe an acceleration gap and FODO quadrupole assembly for a prototype induction accelerator being constructed by FM Technologies for a joint LLNL/LBNL Relativistic-Klystron Two-Beam Accelerator (RK-TBA) technology test-bed experiment. The gap is designed for a $150 \mathrm{kV}$ pulse with a $100 \mathrm{~ns}$ rise time and a $200 \mathrm{~ns}$ flat top provided by the induction cores. A demountable unit consisting of a reentrant geometry with alumina insulator and beam pipe was developed. Achieving the required longitudinal impedance, with the 1-1/8" beam pipe, required careful attention. We examined the electric field distribution, particularly at the opening to the beam pipe and at the insulator triple points. The magnet assemblies are distributed along the beam pipe. Each assembly consists of 12 parallelpipedal ceramic- 8 magnet blocks arranged in a quadrupole Halbach configuration. This assembly is incorporated into a compact $50 \mathrm{~cm}$ induction modulator cell. [1]
\end{abstract}

\section{INTRODUCTION}

The RK-TBA concept uses a 600A relativistic electron drive beam to drive the parallel, lower current main beam. Microwave power at $11.424 \mathrm{GHz}$ is extracted periodically along the beam line for this purpose. Energy is restored to the drive beam using induction accelerators between the extraction locations.

The desired accelerating gradient applied to the drive beam is $300 \mathrm{kV} / \mathrm{m}$. This voltage pulse is to be applied as a 100ns linear rise, 200ns flat top pulse. FM Technologies (FMT) has chosen a half-meter induction cell design, which is therefore designed for $150 \mathrm{kV}$.

The induction cell consists of several components as seen in Figure 1. A modular system approach has been followed. The induction cores, constructed of 2605SC alloy, the outer shell and end-flanges, along with the drive electronics and connections are discussed in a companion paper at this conference. [1]

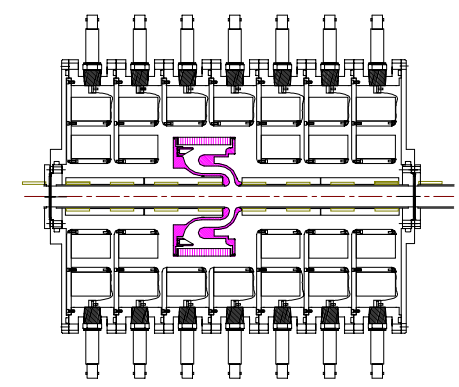

Figure 1: FMT prototype induction cell.
The acceleration gap is attached to the beam pipe and custom UHV flanges are used to provide a demountable connection A unique low impedance design was developed for the gap cavity structure. The gap is integral with the beam transport pipe. The quadrupole FODO magnet assemblies mount the magnets directly against the beam pipe.

\section{DEMOUNTABLE DESIGN}

A demountable, modular design approach for the gap assembly allows replacement of the gap without scrapping the entire module. The acceleration gap is attached to the beam pipe which is welded to custom UHV flanges which are then bolted to the inner face of the module end plates. This provides a demountable gap with only standard mechanical disassembly / reassembly for replacement with gaps of other designs. Alternate gaps can be tested with minimal costs. This is achieved by the use of flanges with two sealing surfaces, a UHV copper gasket face seal and an o-ring pressure seal for the $\mathrm{SF}_{6}$ or oil used around the induction cores as shown in Figure 2 below.

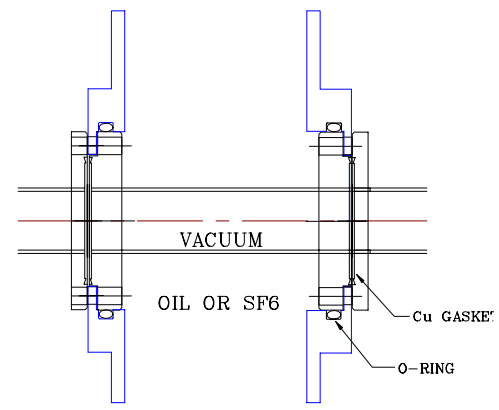

Figure 2: The cavity is mounted with two-seal flanges at each end of the beam pipe.

\section{QUADRUPOLE MAGNET ASSEMBLY}

The use of rectangular parallelpipedal Ceramic-8 magnets arranged in a Halbach configuration allows for an accurate, compact, and inexpensive solution to the construction of the FODO quadrupole transport magnetics. The reduction to $2.5 \mathrm{MeV}$ beam energy and the reduction of the beam pipe diameter to 1-1/8" allowed the use of inexpensive Ceramic- 8 magnets. The required pole tip field is slightly over $1 \mathrm{kG}$. The rectangular cross section magnet blocks are evenly spaced azimuthally. The easy axis orientation was constrained to be parallel to one of the cross-sectional edges, four blocks along the short direction and 8 along the longer direction. 


\subsection{Simulation}

A cross-axis slice of the magnetic flux simulation is shown in Figure 3. The 2D design portion used the PANDIRA code. The 8 "helper" magnets constrain the magnetic flux effectively and allow a significantly more compact design than a traditional 4 block quadrupole design.

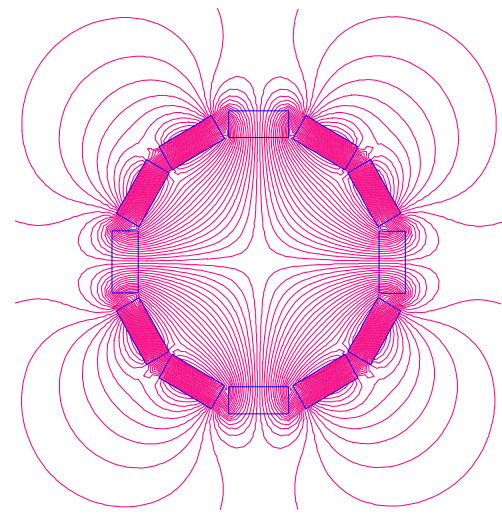

Figure 3: 12-block quadrupole design using PANDIRA

Three-dimensional simulation results using MAG-PC indicate that the linearity integrals are $<0.4 \%$ for $\mathrm{r} / \mathrm{R}<$ $0.3\left(\mathrm{r}_{\mathrm{b}} / \mathrm{R}=4 \mathrm{~mm} / 17.6 \mathrm{~mm}=0.23\right)$.

\subsection{Mounting}

The blocks are bonded into grooves machined on the inner radius of an annular holder as shown in Figure 4. This is split into two half cylinders to allow mounting on the beam pipe after the flanges are welded on.

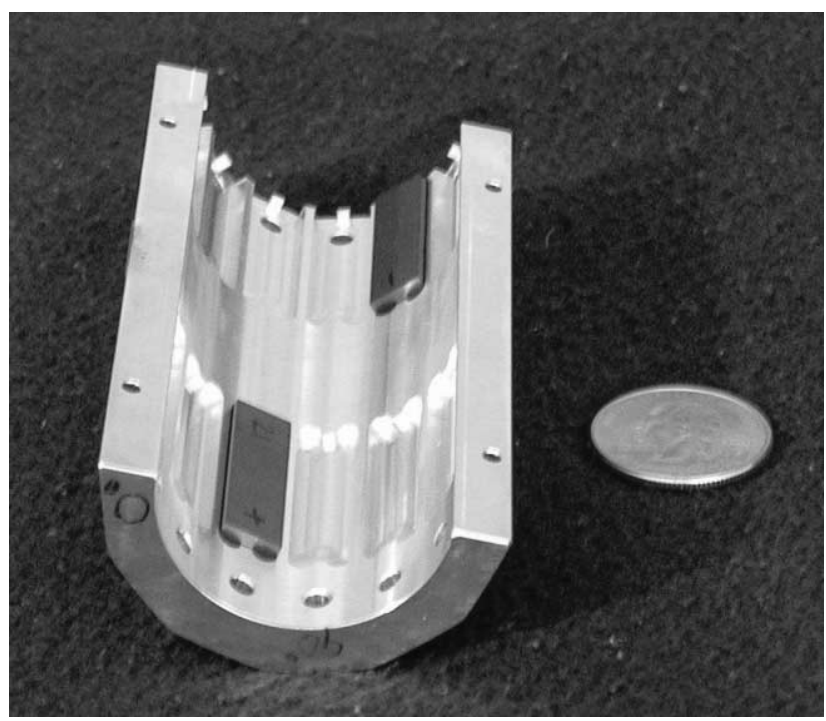

Figure 4: Permanent quadrupole magnets in the block holder. The magnet blocks are held against the beam pipe.

\section{LONGITUDINAL IMPEDANCE}

The longitudinal impedance, $Z_{L}$, of the cavity design must be low to minimize efficiency losses. The design requirement is a power loss to the induction cells of less than $4 \%$ of the $360 \mathrm{MW}$ per two meter of module. This translates for our half-meter module into $\mathrm{Z}_{\mathrm{L}}<3.6 \Omega$ at $11.424 \mathrm{GHz}$.

In order to accommodate scheduling measurements of $\mathrm{Z}_{\mathrm{L}}$, an unoptimized version of the cavity geometry was simulated, constructed, and measured. The cavity was placed inside a microwave absorber lined can. A 1/8" diameter rod extends the length of the system, forming a coaxial line on which a TEM mode is excited. A 5-step binomial matching taper was installed at each end of the beam pipe for matching from the characteristic impedance of the coaxial line to the $50 \Omega$ measurement system.

The correlation between the AMOS simulation and the measurement at $11.424 \mathrm{GHz}$, shown in Figure 5, suggests that $\mathrm{Z}_{\mathrm{L}} \approx 2.1 \Omega$ for an AMOS optimized geometry, shown in Figure 6, would yield a similar correlation.

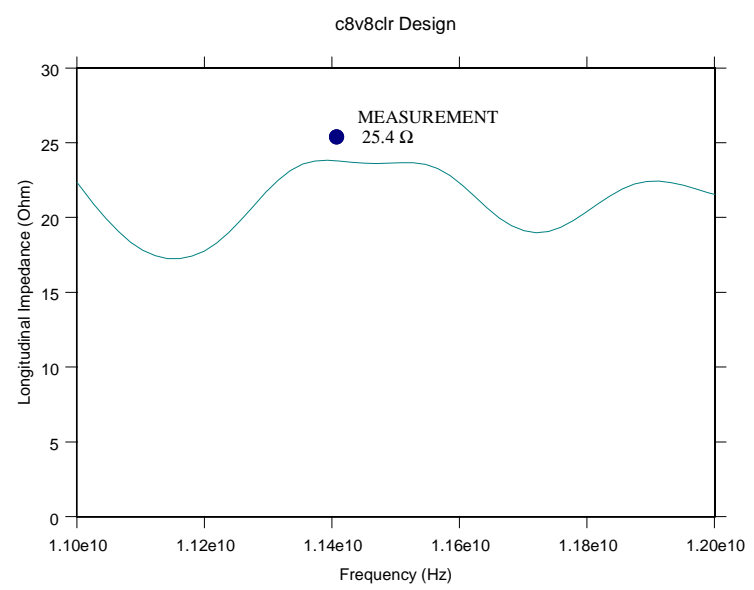

Figure 5: Longitudinal impedance for the prototype geometry.

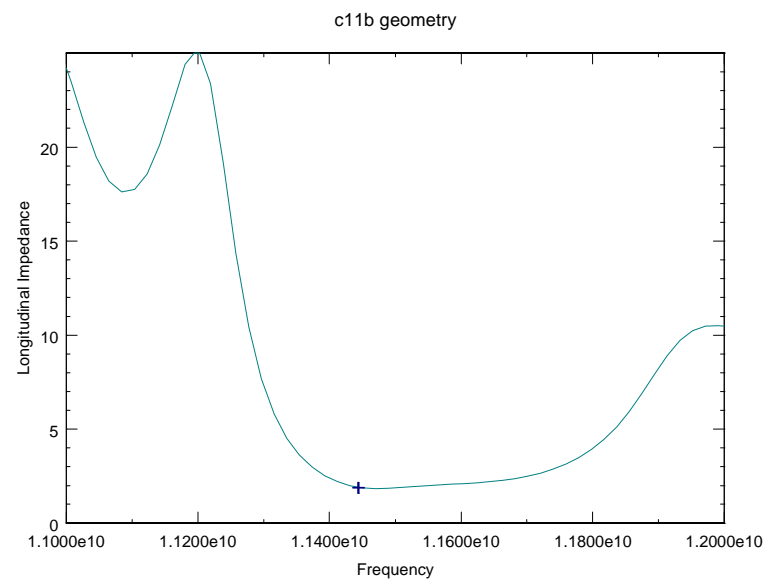

Figure 6: Longitudinal impedance calculated by AMOS for the prototype geometry.

\section{GAP ASSEMBLY}

The reentrant geometry chosen for the gap, shown in Figure 7, provides no line of sight from the beam to the insulator. A simple cylindrical tube for the vacuum insulator was used with an auxiliary insulator ring for 
shaping the field. The angle of the equipotentials in the high field region along the insulator is optimized to sweep electrons away from the insulator. The lower cost of fabrication of the stainless steel electrodes, shown in Figure 8, suggested that the necessary complexities of the geometry be accomplished there.

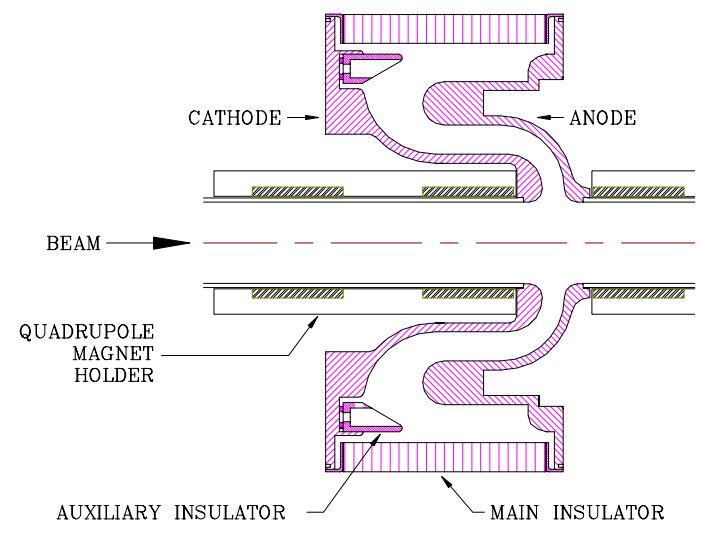

Figure 7: Layout of the gap showing electrodes, insulators, beam pipe and quadrupole magnet assemblies.

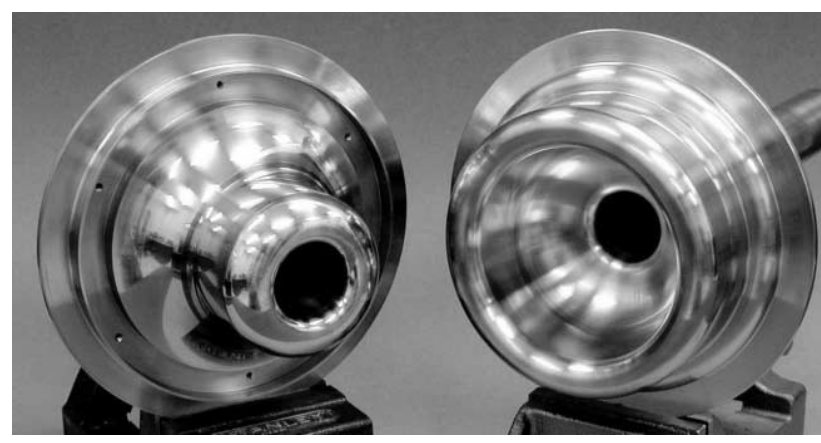

Figure 8: Stainless steel cathode (left) and anode (right).

\section{REFERENCES}

[1] An Efficient Induction Modulator for the Relativistic Klystron Two-Beam Accelerator, S.R. Douglass, T.F. Godlove, and E. Zaidman, this conference. 